\title{
171. Equations of Motion of a Free Particle in the Author's General Relativity as a Non-Holonomic Laguerre Geometry Realized in the Moving Three-Dimensional Cartesian Space
}

\author{
By Tsurusaburo TAKASU
}

(Comm. by Z. Suetuna, M.J.A., Nov. 12, 1954)

The author $[4]^{13}$ has established anew a theory of relativity as a non-holonomic Laguerre geometry realized in the three-dimensional Cartesian space. Although the geometry itself is nothing other than Cartan-Weitzenböck's teleparallelism geometry (Einstein, [2]) keeping the Riemann metric system when it is grasped in 4-dimension, the point of view is different from the Einstein's and has the following fortes ("Vorteile"): (i) it coheres to the physical phenomena in the very places where the phenomena take places: (ii) it is perceptional rather than to be conceptual; (iii) it implies necessities and needs no fundamental assumptions; (iv) it is naive and has an extreme transparency unifying the classical physics, the special relativity (which is in fact a 3-dimensional holonomic Laguerre geometry), the general relativity and the author's necessary unitary field theory, which is non-holonomic parabolic Lie geometry (Takasu, $[5],[6],[7],[8],[9]$ ), and not only that it unifies substantially the gravitation with the electromagnetism in a similar manner ${ }^{2)}$ as the light wave and the electromagnetic wave are unified, but also it admits of fields of (holonomic or non-holonomic) actions of any kind of energy emitted from each particle into unification; ( $v$ ) it admits of the following fundamental principle: "The nonholonomic-Laguerre-geometrical quantum mechanics is obtainable from the Laguerre-geometrical (i.e. special-relativistic) quantum mechanics by replacing the special-relativistic action $d r=c d t$ in the latter by the corresponding non-holonomic-Laguerre-geometrical nonholonomic ${ }^{3)}$ action $d r=E d t$ and the partial derivative operators $\frac{\partial}{\partial \xi}$

1) The ciphers in the square brackets refer to the References at the end of this paper.

2) The more than a dozen unitary field theories hitherto appeared are as for unification all formal ones. They may describe respective ideal cases of phenomena (e.g. if photons emitted by an electron emit energy spherically, the Einstein-Mayer theory describes it. As for the Einstein's new theory [3], Appendix II, see the objection of Prof. C. M $\phi$ ller [10]. As for $g_{\mu \nu}$, see my view [6], p. 264).

3) We can write the differential $d r$ only in the linear sense (e.g. along the geodesic curves of the second kind in the 4-dimension), for $E d t=\omega_{\mu}^{4}(x \lambda) d x^{\mu}$ is not holonomic. 
( $\xi^{l}=$ Cartesian) by their generalizations “ $\frac{\partial}{\omega^{2}}$ ". (vi) Treating of the vector-form $\gamma_{5} \omega^{5}=\gamma_{i} \omega^{l}$, what has proved to be natural for the nonholonomic Laguerre geometry, is physically more powerful than treating of the tensor form $g_{\mu \nu} d x^{\mu} d x^{\nu}$.

In the Prof. Einstein's general relativity, the equations of motion of a free particle are those of the ordinary geodesic curves in 4dimension, while in the author's general relativity, they are those of the geodesic curves of the second kind in 4-dimension, which are homocentric sphere-systems in the three-dimensional non-holonomic Laguerre-geometry (what seems perceptionally to be quite natural).

Sifters for the two sets of equations of motion are of course necessities and experimental data. As for the former the above (iii) shall be referred to.

The purpose of this memoir is to show that some approvable famous data of experiments are supporting the author's general relativity so well as Einstein's.

1. Equations of Motion for a Free Particle. The autoparallel curves of the Cartan-Weitzenböck's teleparallelism have the equations of the form

$$
\frac{d^{2} x^{\lambda}}{d S^{2}}+\Lambda_{\mu \nu}^{\lambda} \frac{d x^{\mu}}{d S} \frac{d x^{\nu}}{d S}=0
$$

where

Owing to the known identities

$$
\begin{aligned}
& \Lambda_{\mu \nu}^{\lambda}=-\omega_{\mu}^{l} \frac{\partial \Omega_{l}{ }^{\lambda}}{\partial x^{\nu}}=\Omega_{n}^{\lambda} \frac{\partial \omega_{\mu}^{n}}{\partial x^{\nu}}, \\
& d s^{2}=-d S^{2}=\omega^{i} \omega^{i}-\omega^{4} \omega^{4},(i=1,2,3), \\
& \omega^{l}=\omega_{\mu}^{l}\left(x^{\lambda}\right) d x^{\mu}, \\
& \omega_{\lambda}^{l} \Omega_{l}^{\mu}=\delta_{\lambda}^{\mu}, \quad \omega_{\lambda}^{l} \Omega_{m}^{\lambda}=\delta_{m}^{l} .
\end{aligned}
$$

$$
\begin{gathered}
\frac{d}{d S} \frac{\omega^{l}}{d S}+\Lambda_{m n}^{l} \frac{\omega^{m}}{d S} \frac{\omega^{n}}{d S}=\omega_{\lambda}^{l}\left(\frac{d^{2} x^{\lambda}}{d S^{2}}+\Lambda_{\mu \nu}^{\lambda} \frac{d x^{\mu}}{d S} \frac{d x^{\nu}}{d S}\right), \\
\Lambda_{m n}^{l}=\Omega_{m}^{\lambda} \Omega_{n}^{\mu} \omega_{\nu}^{l}\left(\Lambda_{\lambda \mu}^{\nu}-\Omega_{n}^{\nu} \frac{\partial \omega_{\lambda}^{\nu}}{\partial x^{\mu}}\right)=0
\end{gathered}
$$

the equation (1.1) may be rewritten as follows:

$$
\frac{d}{d S} \frac{\omega^{2}}{d S}=0
$$

giving rise to

$$
\omega^{l}=a^{l} d S, \quad\left(a^{l}=\text { const. }, \quad a^{i} a^{i}-a^{4} a^{4}=-1\right),
$$

whose finite equations

$$
\xi^{l}=\int \frac{\omega^{l}}{d S} d S=a^{l} S+c^{l}, \quad\left(c^{l}=\text { const. }\right)
$$


represent a geodesic curve of the second kind (autoparallel curves of the teleparallelism) in 4-dimension and a homocentric sphere-system in the 3-dimensional non-holonomic Laguerre geometry.

The author identifies (1.1) (accompanied by (1.2)) i.e. (1.8) i.e. (1.10) with the equations of motion of a free particle. The equation

$$
\frac{d}{d S} \frac{\omega^{l}}{d S}=0 \quad \frac{d^{2} x^{\lambda}}{d S^{2}}+\left\{\begin{array}{c}
\lambda \\
\mu \nu
\end{array}\right\} \frac{d x^{\mu}}{d S} \frac{d x^{\nu}}{d S}=0
$$

is deducible from $\delta \int d S=0$ taking $x^{\lambda}$ and

$$
\begin{array}{l|l}
\omega^{l} & \frac{d x^{\lambda}}{d S}
\end{array}
$$

as variable parameters.

2. The Gravitational Field of an Isolated Particle. As a particular solution [A. S. Eddington [1], p. 85] of the Einstein's equation

$$
R_{\lambda \mu}=0 \text {, }
$$

we take the Schwarzschild's form:

$$
d S^{2}=-\gamma(\rho)^{-1} d \rho^{2}-\rho^{2} d \theta^{2}-\rho^{2} \sin ^{2} \theta d \varphi^{2}+\gamma(\rho) d t^{2},
$$

where

$$
\gamma=1-\frac{2 m}{\rho} .
$$

The solution (2.2) will ultimately be shown to correspond to the field of an isolated particle continually at rest at the origin. Owing to the fact that our non-holonomic Laguerre geometry is realized in the three-dimensional Cartesian space, we may identify $\rho, \theta$ and $\varphi$ with the usual polar coordinates in the Cartesian space (this point differs from the case of Einstein space: cf. A. S. Eddington $[1]$, p. 83).

3. Planetary Orbits. In our case (2.2), the differential equations (1.9) become:

$$
\left\{\begin{array}{rlrl}
\frac{1}{\sqrt{\gamma}} \frac{d \rho}{d S} & =a^{1}, & \rho \frac{d \theta}{d S} & =a^{2}, \\
\rho \sin \theta \frac{d \varphi}{d S} & =a^{3}, & \sqrt{\gamma} \frac{d t}{d S}=a^{4}=k, \text { say. }
\end{array}\right.
$$

Let us consider the case $d \theta=0$ (taking $\theta=\pi / 2$ ) of planetary motion, so that $a^{2}=0$ and the identity $a^{i} a^{i}-a^{4} a^{4}=-1$ becomes:

$$
\left(a^{1}\right)^{2}+\left(a^{3}\right)^{2}-k^{2}=-1 \text {. }
$$

Then (3.1) becomes

$$
\frac{1}{\sqrt{\gamma}} \frac{d \rho}{d S}=a^{1}, \quad \rho \frac{d \varphi}{d S}=a^{3}, \quad \sqrt{\gamma} \frac{d t}{d S}=k .
$$

4) In place of the classical expression $\rho^{2} \frac{d \varphi}{d t}=h$, the expression $\rho^{2} \frac{d \varphi}{d S}=h$ appears in the Einstein's general relativity, to which corresponds $\rho \frac{d \varphi}{d S}=a^{3}$ above. 
Put

$$
h=a^{3} / a^{1} .
$$

Then from (3.1), we obtain

The (3.2) gives

$$
h=\sqrt{\gamma} d \varphi / d \rho .
$$

$$
\frac{1}{\gamma}\left(\frac{d \rho}{d S}\right)^{2}+\left(a^{3}\right)^{2}-k^{2}=-1
$$

namely

$$
\frac{1}{\gamma}\left(\frac{d u}{d \varphi}\right)^{2}+u^{2}=\frac{-1-\left(a^{3}\right)^{2}+k^{2}}{\left(a^{3}\right)^{2}}=\left(\frac{a^{1}}{a^{3}}\right)=\frac{1}{h^{2}},
$$

i.e. by (2.3):

$$
\left(\frac{d u}{d \varphi}\right)^{2}+u^{2}=\frac{1}{h^{2}}+\frac{2 m}{h^{2}} u+2 m u^{3}
$$

where

$$
u=1 / \rho \text {. }
$$

Differentiating (3.6), we obtain [cf. [1], p. 86, (39.61), (39.62)]:

$$
\frac{d^{2} u}{d \varphi^{2}}+u=\frac{m}{h^{2}}+3 m u^{2} \text {. }
$$

In (3.8) we have $3 m u^{2}: \frac{m}{h^{2}}=3 u^{2} h^{2}$ and $1 / h^{2}=\left(-1+k^{2}\right) /\left(a^{3}\right)^{2}-1$, where $\left(a^{3}\right)^{2}=\left(\rho \frac{d \varphi}{d S}\right)^{2}$ is, for ordinary speeds, an extremely small quantity - practically the square of the transverse velocity in terms of the velocity of light. Hence $h^{2}$ is such a small quantity. For example, the above ratio for the earth is 0.00000003 . In practical cases the second term in (3.8) will represent an almost inappreciable correction to the Newtonian orbit

$$
\frac{d^{2} u}{d \varphi^{2}}+u=\frac{m}{h^{2}}
$$

4. The Advance of Perihelion. Notwithstanding the geometry of the author's theory differs from that of Einstein's, the equation (3.8) for the orbit of a planet is of the same form as (39.61) of Eddington [1], p. 86 and so the discussion made by Eddington in Art. 40 there holds equally in the author's case. Thus the data there given support the author's general relativity also.

5. The Deflection of Light. For motion with the speed of light $d S=0$, so that by $\rho \frac{d \varphi}{d S}=a^{3}$ and (3.5), we have $a^{3}=\infty, h=\infty$, and the orbit (3.8) reduces to

$$
\frac{d^{2} u}{d \varphi^{2}}+u=3 m u^{2}
$$


The orbit (5.1) gives the path of ray of light, which coincides with (41.1) of Eddington [1], p. 90. Hence the discussion there made holds equally here. The observed values obtained by five expeditions were as follows:

\begin{tabular}{c|c|c|c|c|c|l}
\hline Observers & Year & Expedition places & $f$ & $N$ & $N^{\prime}$ & \multicolumn{1}{|c}{$E$} \\
\hline Dyson & 1919 & Sobral & $6.3 \mathrm{~m}$ & 7 & 7 & $1.98^{\prime \prime} \pm 0.12^{\prime \prime}$ \\
Eddington & 1919 & Principe & - & 2 & 5 & $1.61^{\prime \prime} \pm 0.30^{\prime \prime}$ \\
Campbell & 1922 & Wallal & 3.6 & 2 & 18 & $1.77^{\prime \prime}$ \\
", & $”$ & " & 5.0 & 4 & $62-85$ & $2.2^{\prime \prime}$ \\
Freundlich & 1929 & Takengon & 8.5 & 4 & 8 & $2.24^{\prime \prime}$ \\
Matsukuma & 1936 & Koshimizu & 5.0 & 1 & 8 & $\left\{\begin{array}{l}2.13^{\prime \prime} \\
1.28^{\prime \prime}\end{array}\right.$
\end{tabular}

Here $f=$ focal distance of the telescope used, $N=$ number of eclipse plates, $N^{\prime}=$ number of stars mapped on a single plate.

These data support the Einstein's theory and the author's theory equally.

6. Displacement of the Fraunhofer Lines. The arguments made by Eddington [1], p. 91-93 hold equally here. The light coming from a stronger (non-holonomic) action field have spectral lines more displaced to the red than those for the light emitted by a resting atom in a weaker (non-holonomic) action field. We mark the quantities for the light coming from the sun with $s$. resting atom near the earth with $e$.

Then in usual notations we have

$$
\frac{\lambda_{s}}{\lambda_{e}}=\frac{\Delta t_{s}}{\Delta t_{e}}=\left(1-\frac{m}{\rho_{e}}\right):\left(1-\frac{m}{\rho_{s}}\right) .
$$

Since $\frac{m}{\rho_{e}}$ is very small compared with 1 ,

$$
\frac{\lambda_{s}}{\lambda_{e}}=\left(1-\frac{m}{\rho_{s}}\right)^{-1}=1+\frac{m}{\rho_{s}}=1+\frac{M_{s}}{R_{s}},
$$

where $M_{s}$ and $R_{s}$ are the mass and the radius of the sun respectively. Hence

$$
\frac{\lambda_{s}-\lambda_{e}}{\lambda_{e}}=\frac{\Delta \lambda}{\lambda}=\frac{M_{s}}{R_{s}}=\frac{1.46}{997000}=0.00000212 .
$$

If we take a star other than the sun and take $M_{s}$ and $R_{s}$ as units of the mass and the radius of the star, we obtain

$$
\frac{\Delta \lambda}{\lambda}=0.00000212 \times \frac{M}{R}=0.634 \mathrm{~km} \times \frac{M}{R},
$$

the Doppler effect being taken into account.

In the case of white dwarf, $M / R$ are large and the observation 
of the shift of the spectral lines are comparatively easy. The chairman W. Adams of the Observatory of Mt. Wilson succeeded in observing about $20 \mathrm{~km} / \mathrm{sec}$. for the white dwarf. His result supported the Einstein's theory of general relativity. Now, notwithstanding the author's geometry adopted is different from Einstein's, the author's result (3.8) coincides with Einstein's, so that Adams' data support equally the author's theory.

\section{References}

[1] A. S. Eddington: The Mathematical Theory of Relativity. Cambridge (1923).

[2] A. Einstein: Neue Möglichkeit für eine einheitliche Feldtheorie von Graviation und Elektrizität, Sitzungsber. d. preuss. Akad. d. Wiss., Phys. -Math. Kl. (1928). Auf die Riemann-Metrik und Fern-Parallelismus gegründete einheitliche Feldtheorie, Math. Ann., 102, 685-697 (1930).

[3] A. Einstein: The Meaning of Relativity, Fourth edition. Princeton (1953).

[4] T. Takasu: The general relativity as a three-dimensional non-holonomic Laguerre geometry of the second kind, its gravitation theory and its quantum mechanics, Yokohama Mathematical Journal, 1, No. 1, 89-104 (1953).

[5] T. Takasu: A combined field theory as a three-dimensional non-holonomic parabolic Lie geometry and its quantum mechanics, Yokohama Mathematical Journal, 1, No. 1, 105-116 (1953).

[6] T. Takasu: A necessary unitary field theory as a non-holonomic parabolic Lie geometry realized in the three-dimensional Cartesian space and its quantum mechanics, Yokohama Mathematical Journal, 1, No. 2, 263-273 (1953).

[7] T. Takasu: A necessary unitary field theory as a non-holonomic parabolic Lie geometry realized in the three-dimensional Cartesian space, Proc. Japan Acad., 29, No. 10, 533-536 (1953).

[8] T. Takasu: A necessary unitary field theory as a non-holonomic parabolic Lie geometry realized in the three-dimensional Cartesian space. II. Proc. Japan Acad., 30, No. 8, 702-708 (1954).

[9] T. Takasu: A necessary unitary field theory as a non-holonomic parabolic Lie geometry realized in the three-dimensional Cartesian space and its quantum mechanics, Yokohama Mathematical Journal, 2, No. 2, 265-273 (1953).

[10] C. Mфller: Review of A. Einstein, The Meaning of Relativity, Fourth edition, Appendix II (1953), Mathematica Scandinavica, 1, No. 2, 317 (1953). 